\title{
1 The RNA helicase DDX5 promotes alveolar rhabdomyosarcoma growth 2 and survival
}

3

4 Alberto Gualtieri ${ }^{1}$, Valerio Licursi ${ }^{2}$ and Chiara Mozzetta ${ }^{1 \# .}$

5

6 1. Institute of Molecular Biology and Pathology (IBPM), National Research Council (CNR)

7 of Italy c/o Department of Biology and Biotechnology “C. Darwin”, Sapienza University, 800185 Rome, Italy

9 2. Department of Biology and Biotechnology "C. Darwin", Sapienza University, 00185 10 Rome, Italy

11

12 \#Correspondence: chiara.mozzetta@uniroma1.it (C.M) 


\section{Abstract}

15 Rhabdomyosarcoma (RMS) is the most common soft-tissue sarcoma of childhood 16 characterized by the inability to exit the proliferative myoblast-like stage. The alveolar

17 fusion positive subtype (FP-ARMS) is the most aggressive and is mainly caused by the 18 expression of PAX3/7-FOXO1 oncoproteins, which are challenging pharmacological 19 targets. Thus, other therapeutic vulnerabilities resulting from gene expression changes 20 are progressively being recognized. Here, we identified the DEAD box RNA helicase 5 21 (DDX5) as a potential therapeutic target to inhibit FP-ARMS growth. We show that DDX5 22 is overexpressed in alveolar RMS cells, demonstrating that its depletion drastically 23 decreases FP-ARMS viability and slows tumor growth in xenograft models. 24 Mechanistically, we provide evidence that DDX5 functions upstream the G9a/AKT 25 survival signalling pathway, by modulating G9a protein stability. Finally, we show that 26 G9a interacts with PAX3-FOXO1 and regulates its activity, thus sustaining FP-ARMS myoblastic state. Together, our findings identify a novel survival-promoting loop in FPARMS and highlight DDX5 as potential therapeutic target to arrest rhabdomyosarcoma growth.

\section{Keywords}




\section{Introduction}

42 Rhabdomyosarcomas (RMS) are aggressive childhood cancers representing the most

43 common soft-tissue sarcomas in pediatric population. Around $60 \%$ of all children and

44 adolescents diagnosed with RMS are cured by currently available multimodal therapies,

45 including surgery, radiation and conventional chemotherapeutic drugs. However, clinical

46 outcomes for patients with high-risk RMS are still poor, emphasizing the urgency to

47 explore more in depth its molecular underpinnings and to devise new effective

48 therapeutic interventions (1).

49 Pediatric RMS are typically divided into two major categories: alveolar (ARMS) and

50 embryonal (ERMS). These two types of RMS are clinically and molecularly different.

51 ERMS are more common, histologically resemble embryonic skeletal muscle, arise early

52 in childhood from head, neck and retroperitoneum, and are typically associated with

53 better prognosis. The genetic profile of ERMS is heterogeneous and is associated with

54 activation of various tumor-promoting signaling pathways and/or loss of tumor

surveillance. ARMS are most common in older children, predominantly involve trunk and

extremities and are generally more aggressive. They typically associate with pathognomonic chromosomal translocations, such as $\mathrm{t}(2 ; 13)$ or $\mathrm{t}(1 ; 13)$ that result in fusion proteins combining the DNA binding domain of PAX3 or PAX7 with the transcriptional activation domain of FOXO1A, which account for the $60 \%$ or $20 \%$ ARMS cases, respectively. The remaining $20 \%$ of ARMS lack molecular evidence of these translocations and are referred to as fusion-negative ARMS (2). Alveolar fusion-positive (FP-ARMS) is the most aggressive subtype, associated with frequent metastasis at the time of diagnosis and limited response to treatment, resulting in poor survival rates. FP-RMS cells are addicted to the oncogenic capacity of PAX3/7FOXO1, which have become very important prognostic markers in the clinics. However, direct targeting of the fusion proteins is still a challenge (3) and other therapeutic 67 vulnerabilities resulting from gene expression changes are being extensively 
investigated in the last years (4-7). In this context, DEAD box RNA helicases appear appealing candidates as potential therapeutic targets, having been implicated in almost every aspect of RNA metabolism, including transcription, pre-mRNA splicing, ribosome biogenesis, transport, translation, and RNA decay (8). In normal myogenesis, the DEAD box helicase 5 (DDX5, also known as p68) is needed for proper differentiation, being part of a multitasking complex that together with the 74 steroid nuclear receptor activator $(S R A)$ long-non coding RNA, BRG1 and MYOD, 75 promotes transcriptional expression of MYOD-target genes (9). Moreover, DDX5 76 cooperates with heterogeneous nuclear ribonucleoprotein (hnRNP) to establish specific 77 splicing subprograms in myoblasts along myogenesis (10), highlighting its multimodal actions in shaping the gene expression programs during cell differentiation. Many studies

79 have detected the overexpression of DDX5 in different human malignancies and confirmed its involvement in tumorigenesis, invasion, proliferation and metastasis (1113). Thus, DDX5 is a potentially valuable diagnostic and prognostic marker in cancer. However, whether DDX5 plays a role in rhabdomyosarcoma pathogenesis has been not addressed yet. Here, we demonstrate that DDX5 is overexpressed in FP-ARMS and that it promotes their survival and growth, both in vitro and in vivo. Mechanistically, we found that DDX5 interacts and cooperates with the lysine methyltransferase G9a to stabilize PAX3-FOXO1 thus sustaining the myoblastic stage of FP-ARMS. 


\section{Results and Discussion}

DDX5 is overexpressed in rhabdomyosarcoma and sustains FP-ARMS survival

To gain insights into a possible role of DDX5 in RMS, we looked at its expression and epigenetic status through the Integrated Rhabdomyosarcoma database of the St. Jude

(Supplemental Figure 1B). Accordingly, DDX5 RNA levels (DDX5 FPKM) did not

significantly differ among normal and RMS samples (Figure 1A, blue bars). By contrast,

proteomic data revealed that DDX5 protein levels were higher than normal cells in all

tested RMS samples (Figure 1A, red bars), which were also accompanied by an

hyperphosphorylated status of DDX5 as compared to normal myoblasts (Figure 1A, circles). Experimental analysis of DDX5 expression, by quantitative real-time PCR (qRTPCR) (Figure 1B) and Western blot (WB) (Figure 1C), on two different FP-ARMS cell lines, $\mathrm{RH} 30$ and $\mathrm{RH} 41$, confirmed DDX5 overexpression in FP-ARMS, as compared to normal human skeletal muscle myoblasts (HSMMs), prompting our interest in investigating its functional role.

To this end, we inhibited DDX5 expression in FP-ARMS cells through small interfering RNA (siRNA)-mediated knock-down (KD). $\mathrm{RH} 30$ and $\mathrm{RH} 41$ cells were transfected with two different siRNA against DDX5 or with scramble siRNAs as control (siCTR). As

110 demonstrated by qRT-PCR (Figure 1D) and western blot (Figure 1E) analysis, both

111 siRNAs efficiently depleted DDX5 in both cell lines. A growth curve performed on both

$112 \mathrm{RH} 30$ (Figure 1F, right panel) and $\mathrm{RH} 41$ (Figure 1F, left panel), demonstrated that

113 DDX5 depletion significantly reduced FP-RMS growth, as compared to control cells 
114 (siCTR). An effect that was visible by morphological inspection upon 72 hrs after

115 treatment, (Figure 1G). Western blot analysis for the apoptotic markers cleaved PARP

116 and cleaved caspase 7 (Figure $1 \mathbf{H})$, clearly demonstrated the induction of programmed

117 cell death in FP-ARMS upon reduction of DDX5 expression. Taken together, these data

118 led us to conclude that overexpression of DDX5 sustains FP-RMS growth and survival.

120 DDX5 promotes AKT signaling stabilizing G9a in FP-RMS

121 To gain further insights into the mechanism behind DDX5 role in sustaining FP-RMS

122 survival, we performed transcriptional profiling by RNA-seq in siDDX5 Rh30 cells, as

123 compared to siCTR-transfected cells (Figure 2A). Notably, enrichment analysis of the

124 differentially expressed genes (DEGs; $p<0.05, F C>1.5$ ) found "regulation of RAS protein

125 signal transduction" among categories enriched in down-regulated transcripts (Figure

126 2B). This evidence caught our attention, as RAS pathway is one of the most de-regulated

127 in both FN-RMS and FP-RMS (16). Its predominant downstream signaling pathways,

128 such as the RAF-MEK (mitogen-activated protein kinase (MAPK) kinase-ERK

129 (extracellular signal-regulated kinase) MAPK pathway and the phosphatidylinositol 3-

130 kinase (PI3-kinase)-AKT-mammalian target of rapamycin (mTOR) pathway), are key to

131 maintain cell growth and proliferation, which is why their inhibition is being tested to arrest

132 cancer cell survival (17), with positive effects reported also in RMS (18). Of note, KEGG

133 analysis of 'RAS signaling' pathway (Figure 2C, left panel) indicated that among its

134 downstream cascades, the 'Akt signaling' pathway was the one affected by DDX5

135 depletion. Accordingly, KEGG on the specific 'PI3K-Akt signaling' pathway highlighted

136 downregulation of $A K T$ (Figure 2C, right panel). AKT is involved in cell survival and

137 proliferation, through the mTOR pathway (19), and in apoptosis inhibition by blocking the

138 FOXO cascade (20). To validate this finding, we performed qRT-PCR for AKT on RH30

139 cell lines after 3 days siDDX5 treatment, demonstrating a significant reduction of AKT

140 mRNA levels as compared to siCTR cells (Figure 2D). Moreover, by western blot 
141 analysis we demonstrated that DDX5 silencing induced a significant reduction of the

142 protein levels of AKT and, consequently, of $\mathrm{p}-\mathrm{AKT}$, the fully activated form of the kinase

143 phosphorylated on Thr 308 and Ser 473 (Figure 2E). These data agree with recent work

144 demonstrating that DDX5 promotes hepatocellular carcinoma cell growth via AKT

145 pathway (21), supporting a similar role for DDX5 in sustaining FP-ARMS growth and

146 survival.

147 It has been recently reported that AKT signaling is also pathogenically activated in FP-

148 ARMS by the lysine methyltransferase G9a (22), an evidence that led us to hypothesize

149 that DDX5 and G9a might exert their function via a common regulatory axis on AKT. To

150 investigate for this potential cooperation, we performed co-immunoprecipitation studies

151 in RH41 cells in control condition (shCTR) and in cells depleted of G9a (by short-hairpin

152 RNA against G9a, shG9a). As shown in Figure 2F, we demonstrated the presence of

153 DDX5 in the immunoprecipitation of G9a, which was reduced in cells with decreased

154 levels of G9a, confirming the specificity of the interaction (Figure 2F). In further support

155 for a possible cooperation between G9a and DDX5, we showed that G9a depletion in

156 FP-ARMS cells phenocopied the downregulation of DDX5. Indeed, shG9a FP-ARMS

157 displayed growth arrest (Supplemental Figure 2A-B); and an increased expression of

158 apoptotic markers (Supplemental Figure 2C). Then, to better investigate the

159 relationship between DDX5 and G9a, we studied their reciprocal modulation. While G9a

160 reduction had no effect on DDX5 protein levels (Figure $\mathbf{2 F}$, input lanes), we observed

161 that depletion of DDX5 led to a significant reduction of G9a protein in $\mathrm{RH} 41$ cells (Figure

162 2G). Since G9a mRNA levels did not significantly decreased after DDX5 silencing

163 (Figure $2 \mathrm{H}$ ), our results suggest that DDX5 regulates G9a post-transcriptionally. In

164 agreement with this idea, it has been recently demonstrated that DDX5 is involved in the

165 alternative splicing of G9a transcripts in spermatogonia (23) a mechanism that has been

166 also shown to affect the stability of different G9a isoforms during neuronal differentiation

167 (24). These results lead to speculate that DDX5 might be involved in the control of G9a 
splicing also in FP-ARMS, likely stabilizing specific isoforms and ultimately affecting G9a

169 protein stability.

170 Taken together, our data indicate that DDX5 function upstream of G9a to promote cell

171 growth and survival, at least in part, via AKT modulation.

172

173 G9a regulates PAX3-FOX01 protein stability thus sustaining FP-RMS myoblastic

\section{4 state}

175 FP-RMS are addicted to the oncogenic capacity of PAX3-FOXO1, which has been firmly

176 implicated in perpetuating the myoblastic proliferative state of RMS cells (7). In light of

177 our results showing that DDX5 and G9a downregulation disrupt this survival-promoting

178 loop, we decided to investigate whether DDX5 and G9a could be involved in the PAX3-

179 FOXO1 modulation.

180 Of note, depletion of both DDX5 (Figure 3A) and G9a (Figure 3B) caused a marked reduction of PAX3-FOXO1 protein; while PAX3-FOXO1 mRNA levels remained unaffected (Supplemental Figure 3A), pointing towards a post-transcriptional regulation. Since PAX3-FOXO1 reduction was observed also upon G9a depletion, a condition in which DDX5 levels are unaffected (Figure 2F), we hypothesized that G9a is the major regulator of PAX3-FOXO1 oncoprotein in this axis. In agreement with this idea, inhibition of G9a enzymatic activity by treatment with two specific small molecule inhibitors, A366 (Figure 3C) and UNC0642 (Figure 3D), exerted similar effects on PAX3FOXO1 levels, further suggesting that G9a mediates PAX3-FOXO1 stability via its enzymatic activity. Accordingly, we found that G9a interacted with PAX3-FOXO1 in two different FP-RMS cell lines (Figure 3E), and this interaction was reduced in cells treated 
195 ARMS cells (Supplemental Figure 3B) induced transcriptional changes inversely

196 correlated by those imposed by PAX3-FOXO1 expression $(26,27)$, as revealed by Gene

197 Set Enrichment Analysis (GSEA) (Figure 3G). Accordingly, transcript levels of known

198 PAX3-FOXO1 target genes were reduced in G9a KD cells (Figure 3H). Further, it has

199 been previously shown that PAX3-FOXO1 activates the RMS master transcription

200 factors (MTFs) MYOD1 and Myogenin, and together with them establishes the

201 epigenome and transcriptional signatures of FP-RMS (7). Strikingly, G9a down-

202 regulation (Figure 3l,L) and pharmacological inhibition (Figure 3M) was sufficient to

203 induce a strong reduction of MYOD1 and Myogenin expression, both at the RNA (Figure

204 3I) and protein (Figure 3L,M) levels. This was confirmed by GSEA that revealed a

205 striking negative correlation of transcripts belonging to the "Myogenesis" hallmark in G9a

206 depleted cells (Supplemental Figure 3C), as compared to control. Taken together,

207 these evidences strongly indicate that G9a activity promotes the PAX3-FOXO1-induced

208 myoblastic RMS stage stabilizing the stability of the oncoprotein.

209

210

\section{DDX5 promotes FP-RMS growth in vivo}

211 Our results identified the existence of a three-component regulation axis in which DDX5 functions upstream of G9a and PAX3-FOXO1 to sustain FP-ARMS survival. To unequivocally demonstrate a role of DDX5 in mediating FP-ARMS tumorigenesis in vivo, we performed xenografts experiments. To this end, we subcutaneously injected both control (shCtr) and DDX5 depleted RH30 cells (shDDX5) into the flanks of BALB/c Nude mice. Consistent with our in vitro data, tumors derived from shDDX5 displayed a significantly reduced growth over time as compared to those derived from control cells (shCtr) (Figure 4A); and excised tumors were much smaller than controls (Figure 4B). Moreover, immunohistological analysis demonstrated that shDDX5 isolated tumors displayed a significant reduced number of proliferating (Ki67+) cells, as compared to 
222 the growth inhibition we previously observed in vitro and demonstrate that DDX5 plays

223 a crucial role in FP-RMS growth in vivo. Moreover, western blot analysis on xenografts-

224 derived protein extracts confirmed our in vitro data, showing a DDX5-dependent

225 expression of G9a and PAX3-FOXO1 in RMS tumors (Figure 4D-E), confirming that

226 DDX5 downregulation also reduces PAKT, AKT and MYOD1 protein (Figure 4E) levels

227 in vivo.

228 In sum, our data identify a major role for DDX5 in sustaining FP-ARMS survival and

229 designate it as a possible novel therapeutic target for rhabdomyosarcoma. DDX5 ha 


\section{Methods}

\section{Cell lines}

233 All cell lines were maintained in a humidified incubator at $37^{\circ} \mathrm{C}$ with $5 \% \mathrm{CO}$. Primary human skeletal muscle myoblasts (HSMMs) were cultured in growth medium (SkGM-2 Bullet Kit, Lonza). HEK293T cells (kindly gifted by Slimane Ait-Si-Ali lab) for the

236 production of lentiviral particles were cultured in Dulbecco's modified Eagle's medium 237 (DMEM) (Sigma-Aldrich, D5671), supplemented with 10\% FBS (Corning, 35-015-CV), 2 $238 \mathrm{mM} \mathrm{L}$-glutamine and $100 \mathrm{U} / \mathrm{ml}$ penicillin/ streptomycin. ARMS cell lines $\mathrm{RH} 30$ and $\mathrm{RH} 41$ 239 were kindly provided by Rossella Rota (Bambino Gesù Children's Hospital, Rome, Italy).

$240 \mathrm{RH} 30$ and $\mathrm{RH} 41$ were maintained in RPMI 1640 with L-glutamine (Sigma-Aldrich, 241 R8758,) supplemented with with 1\% penicillin/streptomycin and 10\% FBS (Corning, 35242 015-CV). Cells were treated with $2 \mu \mathrm{M}$ UNC0642 and $10 \mu \mathrm{M}$ A366 (Sigma-Aldrich, 243 SML1410-25MG) Control cells were treated with equivalent concentrations of DMSO 244 (Sigma-Aldrich). Several first passage aliquots of each cell line were stored in liquid 245 nitrogen for subsequent assays.

\section{Cells transfection}

$248 \mathrm{RH} 30$ and $\mathrm{RH} 41$ cells were transfected with 100nM of human DDX5 specific siRNA 249 (siDDX5 \#1 and siDDX5 \#2, Sigma-Aldrich) or scrambled control siRNA (siCTR) (siRNA 250 universal negative control, Sigma-Aldrich) using Lipofectamine 2000 (Invitrogen) 251 according to the manufacturer's protocol. Transfection with siRNAs was executed when 252 cultured cells reached a confluency of $60 \%$ in 6 well plates. Transfection was carried out 253 according to our adapted protocol in RPMI growth medium for $4-6$ hours at $37^{\circ} \mathrm{C}$. 254 Transfection was then stopped by removing the growth medium and replacing it with 255 RPMI with $10 \%$ fetal bovine serum. RNA or protein were isolated $72 \mathrm{~h}$ post-transfection 256 for all assays. The targeted sense and antisense strands are shown below: 
257 siDDX5 \#1

258 Sense: 5'-AACCGCAACCAUUGACGCCAU-3'

259 Antisense: 5'-AUGGCGUCAAUGGUUGCGGUU-3'

260 siDDX5 \#2

261 Sense: 5'-GGCUAGAUGUGGAAGAUGU-3'

262 Antisense: 5'-ACAUCUUCCACAUCUAGCC-3'

Short hairpin (sh)RNA lentivirus production and cell infections

265 Lentiviruses were produced in HEK293T packaging cells seeded in $100 \mathrm{~mm}$ culture 266 dishes and transfected in 10ml of DMEM medium, using lipofectamine 2000 (Thermo

267 Fisher Scientific), with lentiviral packaging vectors psPAX2 (7 $\mu$; Addgene) and pMD2.G

268 (3,5 $\mu \mathrm{g} ;$ Addgene) and $10 \mu \mathrm{g}$ lentiviral expression constructs shRNA pLKO.1-puro (G9a

269 Mission shRNA, Sigma-Aldrich, TRCN0000115671, NM_025256,); For DDX5

270 knockdown the custom sequence AACCGCAACCAUUGACGCCAU (Sigma-Aldrich

271 DDX5 Mission shRNA plasmid DNA, NM_004396.) was cloned in the pLKO.1-puro

272 vector. The non-silencing shCTR (mission control shRNA plasmid DNA) was purchased

273 from Sigma aldrich. Transfection medium was replaced $24 \mathrm{~h}$ later with new complete

274 DMEM and $48 \mathrm{~h}$ after transfection the lentiviral containing medium was collected, spun

275 to remove cell debris, and the supernatant filtered through a $0.45 \mu \mathrm{m}$ low protein binding

276 filters. Viral aliquots immediately stored at $-80^{\circ} \mathrm{C}$. $\mathrm{RH} 30$ and $\mathrm{RH} 41$ target cells were

277 plated in a $100 \mathrm{~mm}$ dish $\left(1,5 \times 10^{6}\right.$ cells $)$ and, $24 \mathrm{~h}$ later, were infected with lentiviral

278 pLKO.1-puro vectors expressing specific shRNA sequences for $24 \mathrm{~h}$ in the presence of

279 polybrene $(8 \mu \mathrm{g} / \mathrm{ml}$; Sigma-Aldrich). After further $24 \mathrm{hrs}, \mathrm{RH} 30$ and $\mathrm{RH} 41$ cells were

280 selected with $1 \mu \mathrm{g} / \mathrm{ml}$ puromycin (Sigma-Aldrich, P8833) for 3 days. Cells were harvested

281 at different time points for subsequent experiments. All shRNAs were obtained from

282 Sigma Aldrich. 
285 Cells transfected with siRNA DDX5 and cells transduced with lentivirus shRNA G9a were

286 seeded in 6-well plates ( $1.810^{5}$ cells per well) and cell proliferation was evaluated by

287 counting trypsinized cultures at 1,2 and 3 days in $\mathrm{RH} 30$ and $\mathrm{RH} 41$ cells.

289 RNA extraction and Quantitative real time PCR (qRT-PCR)

290 Cells were harvested and centrifuged at $3000 \mathrm{rpm}$ for $5 \mathrm{~min}$ at $4^{\circ} \mathrm{C}$. Supernatant was

291 then removed and cell pellet was resuspended in $1 \mathrm{ml}$ of ice-cold PBS and centrifuged

292 at $2000 \mathrm{rpm}$ for $5 \mathrm{~min}$ at $4^{\circ} \mathrm{C}$. After removing the supernatant, cell pellet was

293 resuspended in $1 \mathrm{~mL}$ of TRI Reagent (Sigma aldrich) and RNA extraction was carried

294 out following manufacturer's protocol. Quantity of RNA samples were assessed with

295 NanoDrop analysis (NanoDrop Technologies). cDNA synthesis was performed using a

296 High Capacity cDNA Reverse Transcription Kits (Applied Biosystems). qRT-PCR was

297 performed with a StepOne plus Real-Time PCR System (Applied Biosystems) to analyze

298 relative gene expression levels using SYBR Green Master mix (Applied Biosystems)

299 following manufacturer indications.

300 PCR amplification was performed as follows: $95^{\circ} \mathrm{C} 5$ minutes, followed by $95^{\circ} \mathrm{C}$ for $10 \mathrm{~s}$, annealing at $60^{\circ} \mathrm{C}$ for $10 \mathrm{~s}$, followed by 45 cycles at $72^{\circ} \mathrm{C}$ for $10 \mathrm{~s}$. Melting curves were generated and tested for a single product after amplification. Expression of each target was calculated using the 2- $\Delta \Delta \mathrm{Ct}$ method and expressed as a relative mRNA expression.

Relative expression values were normalized to the housekeeping gene GAPDH. qRTPCR was done using reaction duplicates and three independent biological replicates were done for each analysis. Error bars indicate the mean \pm standard deviation.

307 The primers we used are:

DDX5,

For:

5'-GCCGGGACCGAGGGTTTGGT-3',

Rev:

5'-

Rev:

5'-GGTCTCCCGCTTGAGGAT-3';

MyoD

For:

5'- 
CCGCCTGAGCAAAGTAAATGA-3'; Rev 5'-GCAACCGCTGGTTTGGATT-3'; RASSF4,

313 For: 5'-AGTCCATTCAGAAGTCGGAGC-3'; Rev: 5'-CCCCAGGCAATGTTGAGGAG-3';

314 PIPOX For: 5'-GGAGCAGTTCTTTCTACCACAC-3'; Rev: 5'

315 TTCCCAGCAGCAGTAATCCA-3'; TFAP2B, For: 5'-TCAATGCATCTCTCCTCGGC-3';

316 Rev:5'-CAGCTTCTCCTTCCACCAGG-

317 3';MRAS,For:TGGCGACCAAACACAATATTCC; Rev: TCTCCCCGCCATTTGGTTTT-

318 3';ABAT,For:5'-CTGCCTCCGGAGAACTTTGT-3';Rev:5'-

319 TTTCCTTGCTCCGGTACCAC-3'; BMP5 For: AATGCCACCAACCACGCTAT Rev: 5'-

320 GCCACATGAGCGTACTACCA-3'; FGFR4, For: 5'-TGGCCGTCAAGATGCTCAAA-3';

321 Rev:5'-GTACAGGGGCCCTTCCTGG-3';GAPDHFor:5'-

322 TCTGGTAAAGTGGATATTGTTGCC-3'; Rev: 5'-CAAGCTTCCCGTTCTCAGCC-

323 3';PAX3-FOXO1, For: 5'-AGACAGCTTTGTGCCTCCAT-3'; Rev: 5'-

324 CTCTTGCCTCCCTCTGGATT-3'; myogenin, For: 5'-TCAACCAGGAGGAGCGTGA-3ì;

Rev: 5'-TGTAGGGTCAGCCGTGAGCA-3'

326

327

\section{Protein extraction and Western blotting}

328 Cells were harvested and centrifuged at $3000 \mathrm{rpm}$ for $5 \mathrm{~min}$ at $4^{\circ} \mathrm{C}$. Supernatant was then removed and cell pellet was resuspended in $1 \mathrm{ml}$ of ice-cold PBS and centrifuged at $2000 \mathrm{rpm}$ for $5 \mathrm{~min}$ at $4^{\circ} \mathrm{C}$. After removing the supernatant, cell pellet was resuspended in lysis RIPA buffer supplemented with protease inhibitor cocktail and phosphatase inhibitors (Roche) and incubated in ice for $30 \mathrm{~min}$. Samples were then sonicated in a water bath for $10 \mathrm{~min}(30 \mathrm{sec}$ ON/ $30 \mathrm{sec}$ OFF) and centrifuged at 15000 rpm for $15 \mathrm{~min}$ at $4^{\circ} \mathrm{C}$. Supernatant was then transferred in a new tube and proteins were 
338 Blot Turbo Transfer system (Bio-Rad Laboratories). Membranes were blocked with 5\%

339 nonfat dried milk in Tris-buffered saline/Tween (TBS-T; 0.1\%) for 1 hours at room

340 temperature with gentle shaking, followed by overnight incubation at $4^{\circ} \mathrm{C}$ with various

341 antibodies. The primary antibodies we used were: DDX5 (Cell Signalling, \#9877), G9a

342 (Cell signalling, \#3306), FOXO1 (Cell Signalling, \#2880), p-AKT (Ser473) (Cell signaling,

343 \#4058), AKT (Cell signaling \#4685) PARP (Cell signaling \#9542), cleaved caspase 7

344 (Cell signaling \#8438), cleaved caspase 9 (Cell signaling \#9505), MYOD (Santa Cruz

345 Biotechnology, C-20), myogenin (DSHB, F5D), GAPDH (Sigma, G9545), a-tubulin

346 (Sigma-Aldrich, T5168). Membranes were then incubated with HRP-conjugated

347 secondary antibodies (IgG-HRP Santa Cruz Biotechnologies) for 1 hour at RT and after

348 incubation the blots were developed in an ECL detection solution (Clarity Max ECL

349 substrate, Bio-Rad Laboratories) and signal was detected using ChemiDoc (BioRad

350 Laboratories).

351

352 Mouse xenograft experiments

353 All animal procedures were approved by Italian Ministry of Health and Istituto Superiore

354 di Sanità; approval number 7FF2C.7-EXT.9.

355 Female Balb/c nude mice (6/7 weeks old) were obtained from (Envigo) and maintained

356 under specific pathogen-free conditions in a temperature- and humidity-controlled

357 environment (Allevamenti Plaisant). $2 \times 10^{6}$ shCTR and shDDX5 RH30 cells were

358 injected subcutaneously into the flank of and, once tumors were palpable, they were

359 measured every other day by measuring 2 diameters (d1 and d2) in right angles using a

360 digital caliper. Total tumor volumes were then calculated by the formula $V=(4 / 3) \pi r 3 ; r=$

$361(\mathrm{~d} 1+\mathrm{d} 2) / 4$. On day 21 after the injections, mice were euthanized and resected tumors

362 were fixed in formalin or immersed in liquid nitrogen and stored at -80 degrees. For

363 western analysis tumors were disrupted with a mortar and pestle, followed by sonication 
364 in RIPA buffer supplemented with proteinase and phosphatase inhibitors (Roche).

365 Formalin-fixed tumor tissues were embedded in paraffin and and sections were stained

366 with hematoxylin and eosin using standard techniques (data not shown). Tissue sections

367 were deparaffinized, rehydrated, and heated at $95^{\circ} \mathrm{C}$ for $20 \mathrm{~min}$ in $\mathrm{pH} 6$ antigen retrieval

368 buffer. Slides were blocked and incubated with Ki67 antibody (Abcam 15580) overnight

369 at $4^{\circ} \mathrm{C}$, then incubated with the secondary antibody (Alexa Fluor 488, Thermo Fisher

370 Scientific). Nuclei were counterstained with DAPI (Sigma). Images were acquired using

371 Axio Observer 443 microscope (ZEISS) and analyzed by ZEN 3.0 (Blue edition)

372 software.

373

374 Co-immunoprecipitation

375 Co-immunoprecipitation was carried out through magnetic separation. $\mathrm{RH} 30$ and $\mathrm{RH} 41$

376 cells were centrifuged at $1200 \mathrm{rpm}$ for $5 \mathrm{~min}$ at $4^{\circ} \mathrm{C}$, resuspended in lysis buffer $(10 \mathrm{mM}$

377 Tris $\mathrm{pH}$ 8, $10 \mathrm{mM} \mathrm{NaCl}, 0.1 \mathrm{mM}$ EDTA $\mathrm{pH}$ 8, $0.1 \mathrm{mM}$ EGTA) with protease inhibitor

378 (Roche) and then incubated on ice for $30 \mathrm{~min}$. A dounce homogenizer was used to 379 mechanically help cell lysis. $10 \%$ NP-40 was added to a final concentration of $0,5 \%$ and 380 then samples were vortexed and incubated on ice 2-3 min. Samples were then 381 centrifuged at $4000 \mathrm{rpm}$ for $5 \mathrm{~min}$ at $4^{\circ} \mathrm{C}$ in order to pellet nuclei. Nuclei were 382 resuspended in nuclei lysis buffer $(20 \mathrm{mM}$ Tris $\mathrm{pH} 8,400 \mathrm{mM} \mathrm{NaCl}, 1 \mathrm{mM}$ EDTA pH 8, $3831 \mathrm{mM}$ EGTA) with protease inhibitor (Roche) and incubated for $10 \mathrm{~min}$ on ice to increase 384 lysis efficiency. Lysates were then sonicated for $10 \mathrm{~min}(30 \mathrm{sec}$ On/30 sec OFF at high 385 intensity) and then centrifuged at top speed for $20 \mathrm{~min}$ at $4^{\circ} \mathrm{C}$. Supernatant, containing 386 nuclear extract (NE), was then transferred into a new tube: $50 \mu \mathrm{l}$ on NE was saved to 387 use as input and to quantify NE concentration. NE was then precleared with $10 \mu$ l protein 388 A/G magnetic beads (Thermo Fisher Scientific), washed with IP lysis buffer (50 mM Tris $389 \mathrm{pH} 8,150 \mathrm{mM} \mathrm{NaCl}, 1 \mathrm{mM}$ EDTA pH 8, $1 \mathrm{mM} \mathrm{EGTA)} \mathrm{for} 2 \mathrm{hrs}$ at $4^{\circ} \mathrm{C}$ on rotating wheel. 
390 After preclearing, NE were diluted 1:5 in IP buffer with protease inhibitor (Roche). NE

391 was incubated overnight at $4^{\circ} \mathrm{C}$ on rotating wheel with 10 ug of G9a antibody (Abcam,

392 ab185050). The following day we added pre-blocked protein A/G magnetic beads

393 (Thermo Fisher Scientific) to each sample and incubated for $2 \mathrm{hrs}$ at $4^{\circ} \mathrm{C}$ on rotating

394 wheel. Samples were then washed six times, one wash every 5 min, with IP buffer. At

395 the end, beads were separated on a magnet and the immunocomplexes were

396 resuspended with IP buffer and LSB buffer (Biorad Laboratories) for further analysis.

397

398 RNA-Sequencing

399 RNAseq in siDDX5 FP-RMS

400 Total RNA was extracted and quantified as previously described. RNA-Seq libraries

401 preparation and sequencing was performed by the IGA Technology Services (Udine,

402 Italy) using the Illumina TruSeq Stranded mRNA Kit (Illumina, San Diego, CA) according

403 to manufacturer's instructions. The final libraries for paired-end sequencing of 150 base

404 pairs were carried out on an Illumina NovaSeq6000 (Illumina, San Diego, CA) with an

405 average of 55 million of reads per sample. Processing raw data for both format

406 conversion and de-multiplexing were performed by Bcl2Fastq version 2.20 of the Illumina

407 pipeline. Reads quality was evaluated using FastQC (version 0.11.8, Babraham Institute

408 Cambridge, UK) tool, then adapter sequences were masked with Cutadapt version 1.11

409 from raw fastq data using the following parameters: --anywhere (on both adapter

410 sequences) --overlap 5 --times 2 --minimum-length 35 --mask-adapter.

411 Reads were mapped to the human Ensembl GRCh38 transcriptome index (release 96)

412 using kallisto (version 0.46.0) (28). The following flags were used for kallisto: $-b 30$--

413 bias. Gene-level normalization and differential gene expression analysis were performed

414 using Bioconductor (29) $\mathrm{R}$ (version 3.6.2) (R Core Team, 2015) package DESeq2

415 version 1.26 (30) accounting for the presence of batch effects. The figures were obtained 
416 using the $R$ environment with package ggplot2 version 3.3 .0 and pheatmap version

$417 \quad 1.0 .12$

418

\section{RNAseq in shG9a FP-RMS}

420 RNA-seq libraries from total RNA (100 ng) from each sample were prepared using

421 QuantSeq 3'mRNA-Seq Library prep kit (Lexogen, Vienna, Austria) according to

422 manufacturer's instructions, at Telethon Institute of Genetics and Medicine (TIGEM). The

423 amplified fragmented cDNA of $300 \mathrm{bp}$ in size were sequenced in single-end mode using

424 the NextSeq500 (Illumina) with a read length of $75 \mathrm{bp}$. Reads quality was evaluated

425 using FastQC (version 0.11.8, Babraham Institute Cambridge, UK) tool and was trimmed

426 using TrimGalore software to remove adapter and low-quality bases $(Q<20)$. Then

427 reads were mapped to the human Ensembl GRCh38 build reference genome using 428 STAR version 2.5.0a (31) using Gene annotations corresponding to the Ensembl

429 annotation release 96 which was used to build a transcriptome index and provided to 430 STAR during the alignment.

431 The same gene annotations were used to quantify the gene-level read counts using HTSeq-count version 0.8 .0 (32) script, subsequently the data normalization and differential analysis for gene expression were performed using Bioconductor (29) R package edge $R$ version 3.28 (33).

\section{Gene set enrichment analysis}

437 In order to understand biological meaning of the differentially expressed genes the 438 resulting filtered (Benjamini-Hochberg false discovery rate (FDR) adjusted for multiple 439 hypothesis testing $p$-value $<0.05)$ genes were clustered by functional annotation using 440 Bioconductor $R$ package clusterProfiler version 3.14 (34) with annotation of Gene 441 Ontology Database (35) and with annotation of Kyoto Encyclopedia of Genes and 442 Genomes (KEGG) (36) for pathways. Gene Set Enrichment Analysis (GSEA) (37) with 
443 pre-ranked, "classic" mode with 10,000 permutations was used to assess the enrichment

444 of the gene profile of siDDX5 or shG9a samples compared to control samples in the

445 curated "hallmark" and "C2" gene set collections (BROAD molecular signature database,

446 MSigDb version 6.2).

447

448 Data availability

449 RNA-Seq data accompanying this paper are available through NCBI's Gene Expression

450 Omnibus (GEO) repository, under accession number GSE152358 and GSE152359.

451

\section{Statistical analysis}

453 Data were analyzed using Prism (version 6.0; GraphPad Software Inc.), and images 454 were compiled in Photoshop (version 6.0; Adobe Systems). Results are presented as 455 mean \pm SD from at least 3 independent experiments. Statistical analysis was conducted 456 using an unpaired Student's t-test, one-way ANOVA or 2-way ANOVA. $P$ value of less 457 than 0.05 was considered statistically significant. ${ }^{\star} P<0.05 ;{ }^{* \star} P<0.01 ;{ }^{\star \star \star} P<0.001$; ${ }^{* * * *} P<0.0001$

459

\section{Author Contributions}

461 A.G. performed all the experiments, collected and analysed data and prepared figures.

462 V.L. performed bioinformatic analyses. C.M. conceived, supervised the project and wrote 463 the manuscript. All authors discussed results, reviewed and edited the manuscript.

\section{Acknowledgements}

466 This work was supported by the Italian Association for Cancer Research (AIRC;

467 MyFIRST grant n.18993) and the CNCCS (Collection of National Chemical Compounds 468 and Screening Center). We would like to thank Marco Pezzullo and Cristiano De Stefanis 
469 (core facilities, Bambino Gesù Children's Hospital, Rome, Italy) for the technical FFPE

470 tissues preparation.

471

472 Declaration of Interests

473 The authors declare no competing interests.

\section{$474 \quad$ References}

475

476

477

478

479

480

481

482

483

484

485

486

487

488

489

490

491

492

493

494

495

496

497

498

499

500

501

502

503

504

505

506

507

508

509

1. Hettmer S, Li Z, Billin AN, Barr FG, Cornelison DD, Ehrlich AR, et al. Rhabdomyosarcoma: current challenges and their implications for developing therapies. Cold Spring Harb Perspect Med. 2014;4(11):a025650.

2. El Demellawy D, McGowan-Jordan J, de Nanassy J, Chernetsova E, and Nasr A. Update on molecular findings in rhabdomyosarcoma. Pathology. 2017;49(3):238-46.

3. Wachtel M, and Schafer BW. PAX3-FOXO1: Zooming in on an "undruggable" target. Semin Cancer Biol. 2018;50:115-23.

4. Seki M, Nishimura R, Yoshida K, Shimamura T, Shiraishi Y, Sato Y, et al. Integrated genetic and epigenetic analysis defines novel molecular subgroups in rhabdomyosarcoma. Nat Commun. 2015;6:7557.

5. Kohsaka S, Shukla N, Ameur N, Ito T, Ng CK, Wang L, et al. A recurrent neomorphic mutation in MYOD1 defines a clinically aggressive subset of embryonal rhabdomyosarcoma associated with PI3K-AKT pathway mutations. Nat Genet. 2014;46(6):595-600.

6. Shern JF, Chen L, Chmielecki J, Wei JS, Patidar R, Rosenberg M, et al. Comprehensive genomic analysis of rhabdomyosarcoma reveals a landscape of alterations affecting a common genetic axis in fusion-positive and fusionnegative tumors. Cancer Discov. 2014;4(2):216-31.

7. Gryder BE, Yohe ME, Chou HC, Zhang X, Marques J, Wachtel M, et al. PAX3FOXO1 Establishes Myogenic Super Enhancers and Confers BET Bromodomain Vulnerability. Cancer Discov. 2017;7(8):884-99.

8. Linder $P$, and Jankowsky E. From unwinding to clamping - the DEAD box RNA helicase family. Nat Rev Mol Cell Biol. 2011;12(8):505-16.

9. Caretti G, Schiltz RL, Dilworth FJ, Di Padova M, Zhao P, Ogryzko V, et al. The RNA helicases p68/p72 and the noncoding RNA SRA are coregulators of MyoD and skeletal muscle differentiation. Dev Cell. 2006;11(4):547-60.

10. Dardenne E, Polay Espinoza M, Fattet L, Germann S, Lambert MP, Neil $H$, et al. RNA helicases DDX5 and DDX17 dynamically orchestrate transcription, miRNA, and splicing programs in cell differentiation. Cell Rep. 2014;7(6):1900-13.

11. Yang L, Lin C, and Liu ZR. Phosphorylations of DEAD box p68 RNA helicase are associated with cancer development and cell proliferation. Mol Cancer Res. 2005;3(6):355-63.

12. Shin S, Rossow KL, Grande JP, and Janknecht R. Involvement of RNA helicases p68 and p72 in colon cancer. Cancer Res. 2007;67(16):7572-8. 
510

511

512

513

514

515

516

517

518

519

520

521

522

523

524

525

526

527

528

529

530

531

532

533

534

535

536

537

538

539

540

541

542

543

544

545

546

547

548

549

550

551

552

553

554

555

13. Nyamao RM, Wu J, Yu L, Xiao X, and Zhang FM. Roles of DDX5 in the tumorigenesis, proliferation, differentiation, metastasis and pathway regulation of human malignancies. Biochim Biophys Acta Rev Cancer. 2019;1871(1):85-98.

14. Stewart E, McEvoy J, Wang H, Chen X, Honnell V, Ocarz M, et al. Identification of Therapeutic Targets in Rhabdomyosarcoma through Integrated Genomic, Epigenomic, and Proteomic Analyses. Cancer Cell. 2018;34(3):411-26 e19.

15. Ernst J, and Kellis M. ChromHMM: automating chromatin-state discovery and characterization. Nat Methods. 2012;9(3):215-6.

16. Skapek SX, Ferrari A, Gupta AA, Lupo PJ, Butler E, Shipley J, et al. Rhabdomyosarcoma. Nat Rev Dis Primers. 2019;5(1):1.

17. Cox AD, Fesik SW, Kimmelman AC, Luo J, and Der CJ. Drugging the undruggable RAS: Mission possible? Nat Rev Drug Discov. 2014;13(11):828-51.

18. Yohe ME, Gryder BE, Shern JF, Song YK, Chou HC, Sindiri S, et al. MEK inhibition induces MYOG and remodels super-enhancers in RAS-driven rhabdomyosarcoma. Sci Transl Med. 2018;10(448).

19. Laplante M, and Sabatini DM. mTOR signaling in growth control and disease. Cell. 2012;149(2):274-93.

20. Zhang X, Tang N, Hadden TJ, and Rishi AK. Akt, FoxO and regulation of apoptosis. Biochim Biophys Acta. 2011;1813(11):1978-86.

21. Xue Y, Jia X, Li L, Dong X, Ling J, Yuan J, et al. DDX5 promotes hepatocellular carcinoma tumorigenesis via Akt signaling pathway. Biochem Biophys Res Commun. 2018;503(4):2885-91.

22. Bhat AV, Palanichamy Kala M, Rao VK, Pignata L, Lim HJ, Suriyamurthy $S$, et al. Epigenetic Regulation of the PTEN-AKT-RAC1 Axis by G9a Is Critical for Tumor Growth in Alveolar Rhabdomyosarcoma. Cancer Res. 2019;79(9):2232-43.

23. Legrand JMD, Chan AL, La HM, Rossello FJ, Anko ML, Fuller-Pace FV, et al. DDX5 plays essential transcriptional and post-transcriptional roles in the maintenance and function of spermatogonia. Nat Commun. 2019;10(1):2278.

24. Fiszbein A, Giono LE, Quaglino A, Berardino BG, Sigaut L, von Bilderling C, et al. Alternative Splicing of G9a Regulates Neuronal Differentiation. Cell Rep. 2016;14(12):2797-808.

25. Cornett EM, Ferry L, Defossez PA, and Rothbart SB. Lysine Methylation Regulators Moonlighting outside the Epigenome. Mol Cell. 2019;75(6):1092-101.

26. Ren YX, Finckenstein FG, Abdueva DA, Shahbazian V, Chung B, Weinberg KI, et al. Mouse mesenchymal stem cells expressing PAX-FKHR form alveolar rhabdomyosarcomas by cooperating with secondary mutations. Cancer Res. 2008;68(16):6587-97.

27. Davicioni E, Finckenstein FG, Shahbazian V, Buckley JD, Triche TJ, and Anderson MJ. Identification of a PAX-FKHR gene expression signature that defines molecular classes and determines the prognosis of alveolar rhabdomyosarcomas. Cancer Res. 2006;66(14):6936-46.

28. Bray NL, Pimentel H, Melsted P, and Pachter L. Near-optimal probabilistic RNAseq quantification. Nat Biotechnol. 2016;34(5):525-7.

29. Gentleman RC, Carey VJ, Bates DM, Bolstad B, Dettling M, Dudoit S, et al. Bioconductor: open software development for computational biology and bioinformatics. Genome Biol. 2004;5(10):R80. 
30. Love $\mathrm{MI}$, Huber $\mathrm{W}$, and Anders S. Moderated estimation of fold change and dispersion for RNA-seq data with DESeq2. Genome Biol. 2014;15(12):550.

558 Dobin A, Davis CA, Schlesinger F, Drenkow J, Zaleski C, Jha S, et al. STAR: ultrafast universal RNA-seq aligner. Bioinformatics. 2013;29(1):15-21.

560 Anders S, Pyl PT, and Huber W. HTSeq--a Python framework to work with highthroughput sequencing data. Bioinformatics. 2015;31(2):166-9.

562 Robinson MD, McCarthy DJ, and Smyth GK. edgeR: a Bioconductor package for differential expression analysis of digital gene expression data. Bioinformatics. 2010;26(1):139-40.

565

34. Yu G, Wang LG, Han Y, and He QY. clusterProfiler: an R package for comparing biological themes among gene clusters. OMICS. 2012;16(5):284-7.

567

35. Ashburner M, Ball CA, Blake JA, Botstein D, Butler H, Cherry JM, et al. Gene ontology: tool for the unification of biology. The Gene Ontology Consortium. Nat Genet. 2000;25(1):25-9.

570

36. Ogata H, Goto S, Sato K, Fujibuchi W, Bono H, and Kanehisa M. KEGG: Kyoto Encyclopedia of Genes and Genomes. Nucleic Acids Res. 1999;27(1):29-34.

37. Subramanian A, Tamayo P, Mootha VK, Mukherjee S, Ebert BL, Gillette MA, et al. Gene set enrichment analysis: a knowledge-based approach for interpreting genome-wide expression profiles. Proc Natl Acad Sci U S A. 2005;102(43):1554550. 
bioRxiv preprint doi: https://doi.org/10.1101/2020.07.08.194092; this version posted July 10, 2020. The copyright holder for this preprint (which was not certified by peer review) is the author/funder, who has granted bioRxiv a license to display the preprint in perpetuity. It is made available under aCC-BY-NC-ND 4.0 International license. 
bioRxiv preprint doi: https://doi.org/10.1101/2020.07.08.194092; this version posted July 10, 2020. The copyright holder for this preprint (which was not certified by peer review) is the author/funder, who has granted bioRxiv a license to display the preprint in perpetuity. It is

A

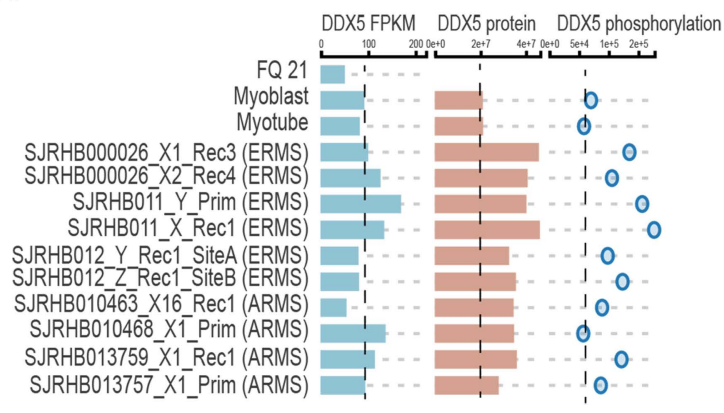

D
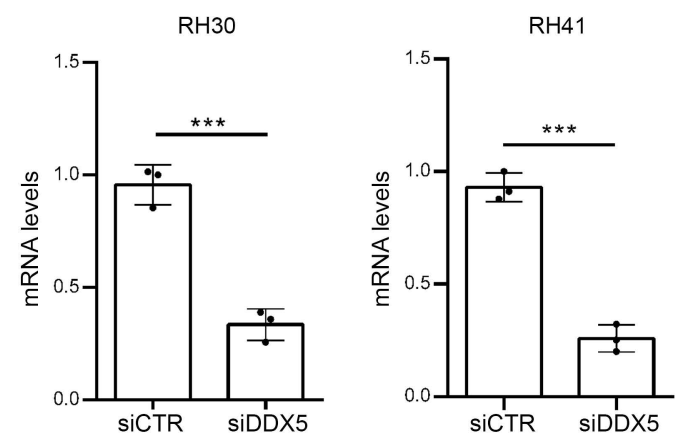

B

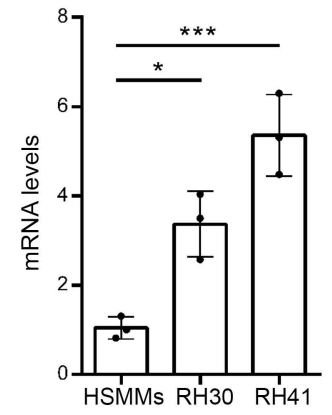

C

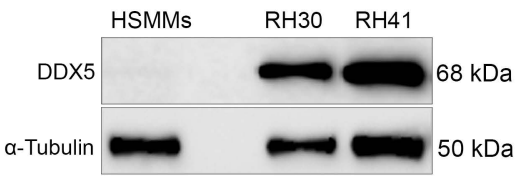

E

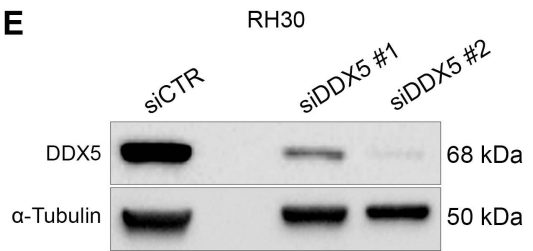

$\mathbf{F}$

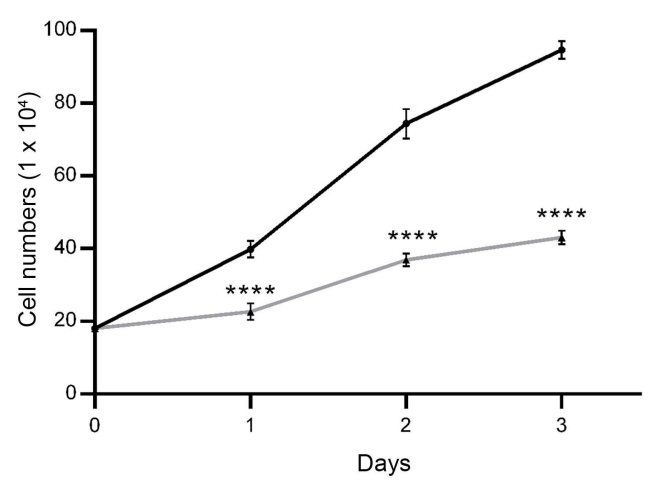

G

$\mathrm{RH} 30$

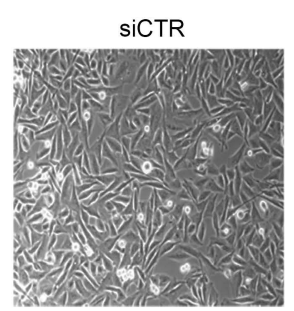

siDDX $\# 1$

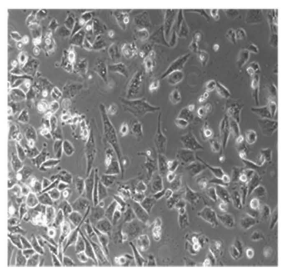

$\operatorname{siDDX5} \# 2$

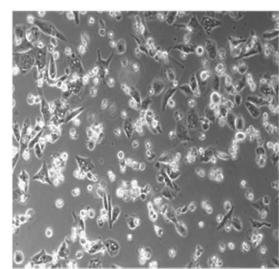

RH41

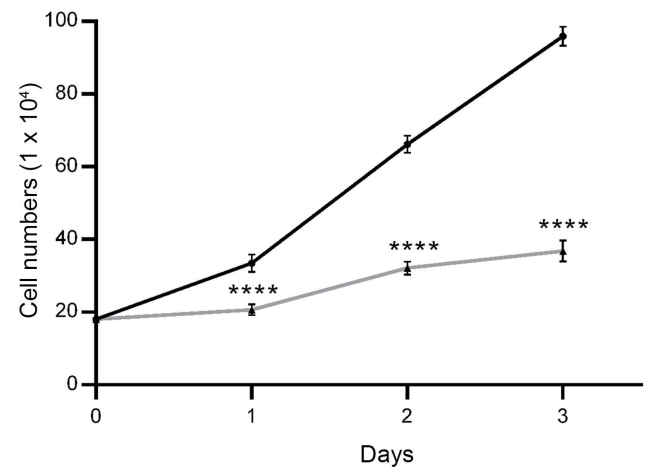

H

$\mathrm{RH} 30$

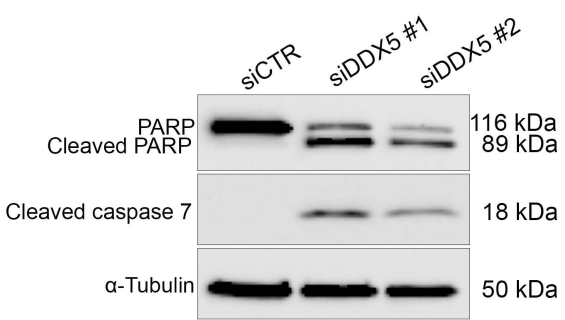

Figure 1. DDX5 is overexpressed in alveolar rhabdomyosarcoma and promotes FP-ARMS survival

(A) RNA (FPKM; blue bars) and protein levels (red bars), together with phosphorylation status (blue circles) of DDX5 in orhtotipic RMS patient-derived xenografts (PDX), as compared to normal myoblasts and myotubes. Data derive from https://pecan.stjude.cloud/proteinpaint/study/RHB2018 (14).

(B) Histogram showing the relative mRNA expression levels of DDX5 in RH30 and RH41 cell lines and human skeletal muscle myoblasts (HSMMs). Transcription values were assessed by qRT-PCR and normalized to GAPDH. Graph represents mean +/- SD from n=3 independent experiments.

(C) Representative western blot analysis for DDX5 in RH30 and RH41 cell lines and HSMMs. Q-tubulin was used as a loading control.

(D) qRT-PCR analysis of DDX5 mRNA levels in RH30 (left) and RH41 (right) cell lines, after siCTR and siDDX5 treatment. Graphs show mean +/- SD from $n=3$ independent experiments. (E) Western blot analysis for DDX5 in RH30 cells treated with siCTR, and two different sequences for DDX5 (siDDX5 \#1 and \#2). Normalization with $\alpha$-tubulin. (F) Cells growth curves after siDDX5 treatment. Cells were counted 1,2 and 3 days after treatment. Graphs show mean +/- SD from $n=3$ independent experiments. (G) Representative phase contrast images of RH30 cells 3 days after siDDX5 treatment. $(\mathbf{H})$ Western blot analysis for indicated proteins performed on RH30 after siDDX5 and siCTR treatment. $\alpha$-tubulin was used as control of loading normalization. Statistical significance has been assessed in (B) by one-way ANOVA with Bonferroni multiple comparisons test. ${ }^{*} p<0.05 ;{ }^{* * *} p$ $<0.001$; (D) by an unpaired Student's t-test; ${ }^{* * *} p<0.001$; and $(\mathbf{F})$ with 2 -way anova with Sidak's multiple comparison test, . ${ }^{* * *} p<0.001$ test. 


\section{FIGURE 2}

(which was not certified by peer review) is the author/funder, wBo has granted bioRxiv a license to display the preprint in perpetuity. It is

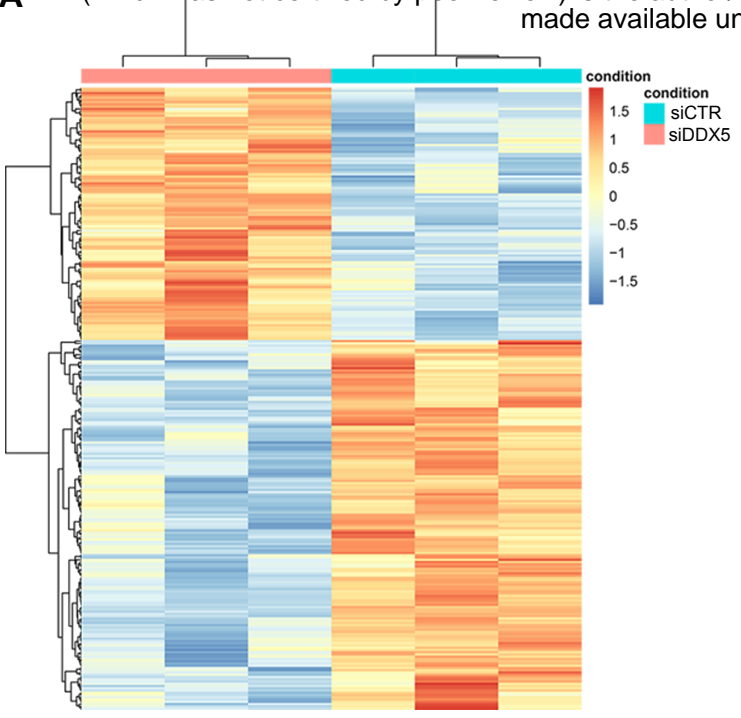
has granted bioRxiv a license to displ
Y-NC-ND 4.0 International license.

RAS SIGNALING PATHWAY

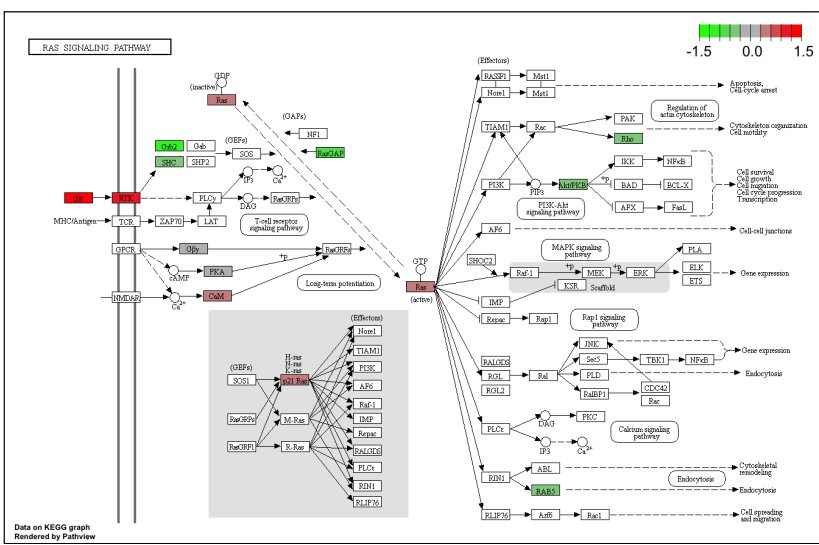

D

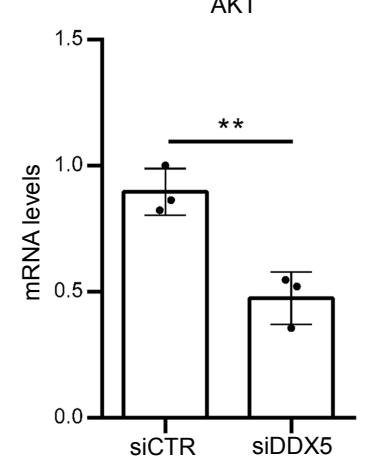

G

$\mathrm{RH} 41$

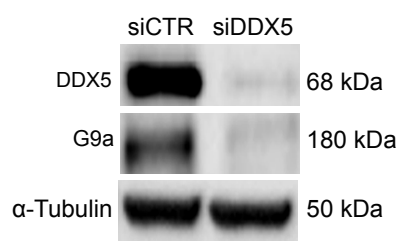

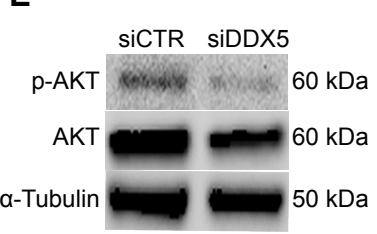

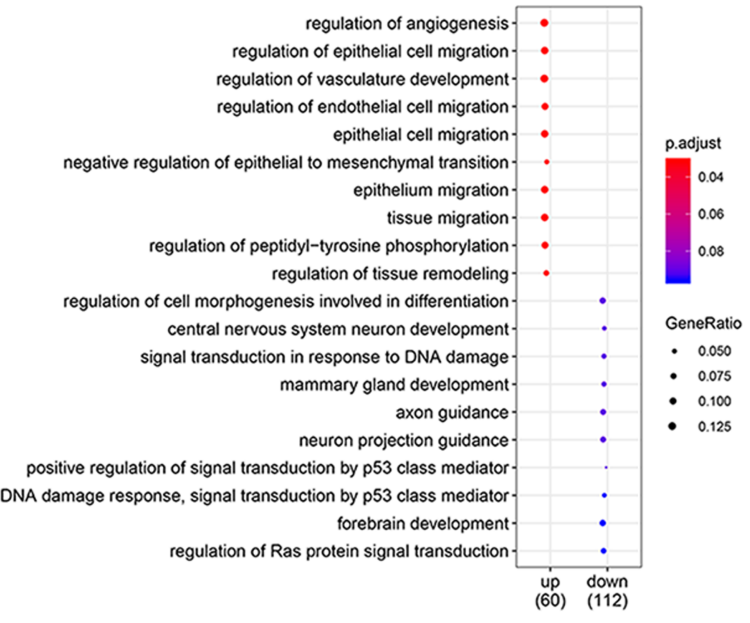

PI3K-AKT SIGNALING PATHWAY

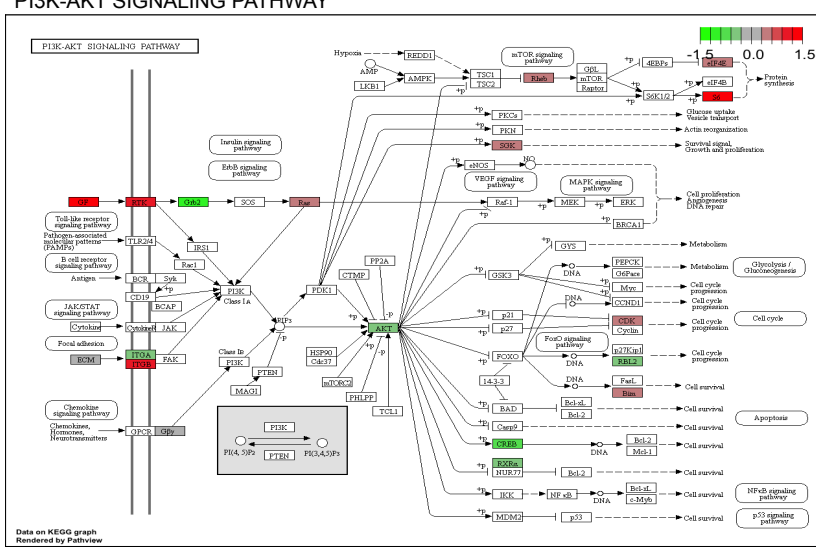

$\mathbf{F}$

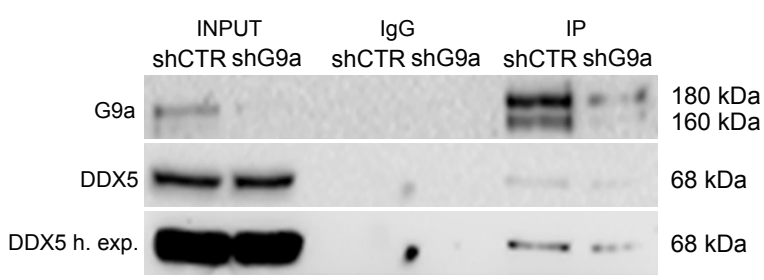

H

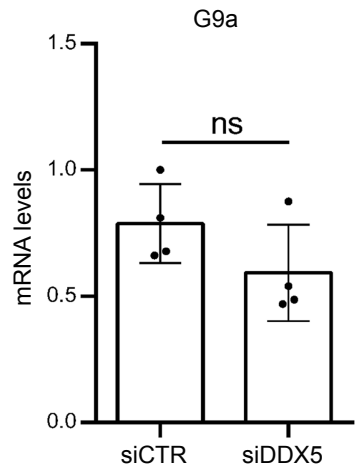

Figure 2 DDX5 interacts with G9a and regulates G9a-AKT signaling

(A) Heatmap plot of hierarchical clustering of all differentially expressed genes (DEGs). The $X$ axis represents the two compared samples (siCTR and siDDX5). The $Y$ axis represents DEGs. The color (from blue to red) represents gene expression intensity from low to high. Red indicates upregulated and blue represents downregulated genes. DEGs were selected according to FC > 1,5 e padj < 0.05. (B) Dot plot showing the up and down regulated GO terms of biological processes identified. The size of the dot is based on gene count enriched in the pathway, and the color of the dot shows the pathway enrichment significance. (C) KEGG analysis of Ras (left) and PI3K-AKT (right) signaling pathways.(D) AKT mRNA levels quantified by qRT-PCR after DDX5 silencing in RH30 cells, as compared to siCTR cells. Transcription values were assessed by qRT-PCR and normalized to GAPDH. Graph represents mean $+/-$ SD from $n=3$ independent experiments. Statistical significance assessed by unpaired Student's t-test; $\left.{ }^{* *} p<0.01\right)$. (E) Western blot analysis of p-AKT and AKT in siCTR and siDDX5 RH30 cells. Normalization with $\alpha$-tubulin (F) Representative western blot analysis of the indicated proteins in G9a immunoprecipitation in nuclear extracts of control (shCTR) and shG9a-treated RH41 cells (last two right lanes). Equal amounts of nuclear extracts were immunoprecipitated with IgG as negative control. Inputs are shown on the left. (G) Western blot analysis for G9a and DDX5 in RH30 cells treated with siCTR and siDDX5. a-tubulin was used as loading control. (H) qRT-PCR for G9a in siCTR and siDDX5 RH30 cells. Graph shows the mean +/- SD value derived from $n=4$ independent experiments. Statistical significance has been assessed by an unpaired Student's t-test; $p>0,05$ (no statistical significance, ns). 

bioRxiv preprint doi: https://doi.org/10.1101/2020.07.08.194092; this version posted July 10,2020 . The copyright holder for this preprint
(which was not certified by peer review) is the author/funder, who has granted bioRxiv a license to display the preprint in perpetuity. It is $\mathrm{RH} 30$
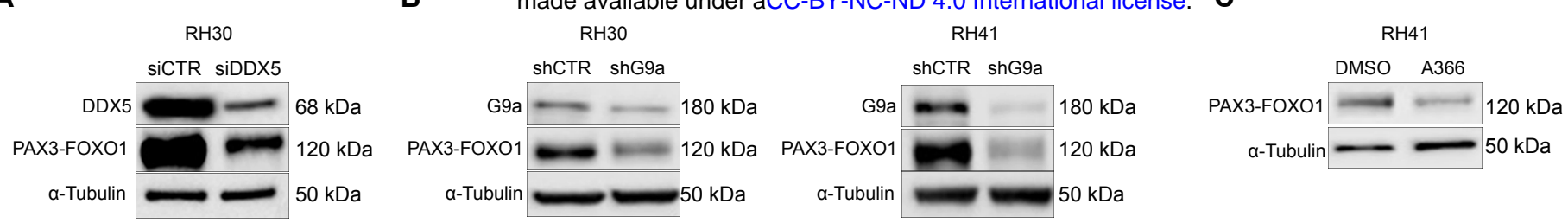

D

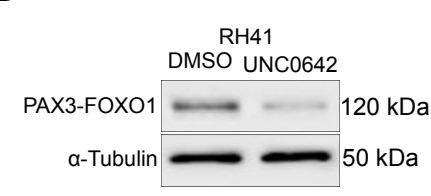

$\mathbf{F}$

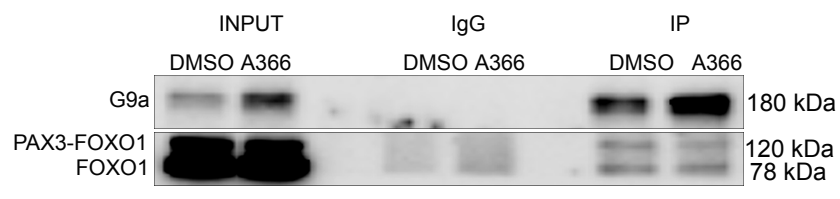

H

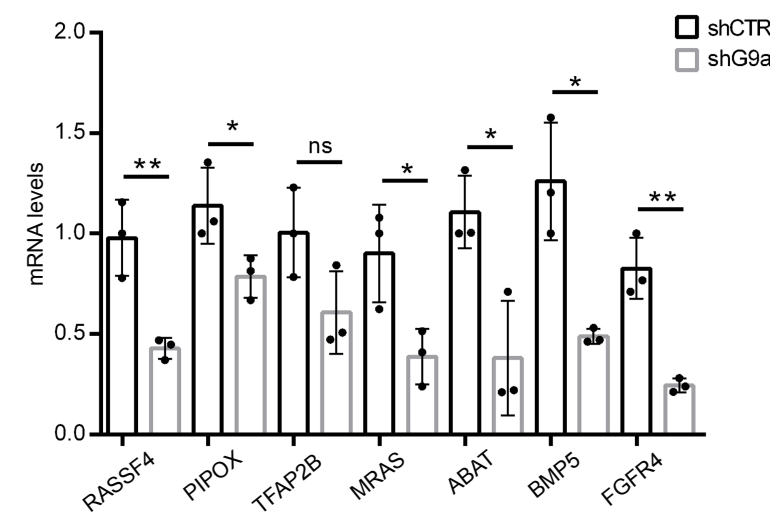

I

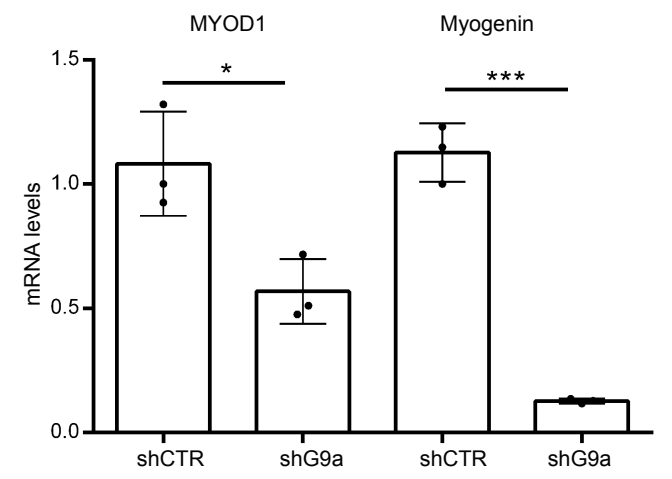

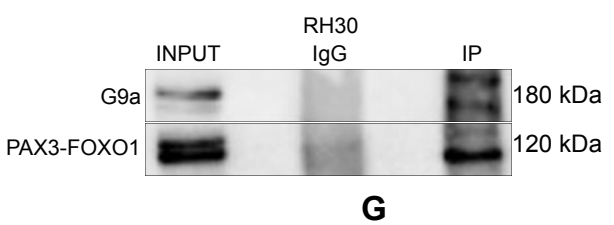

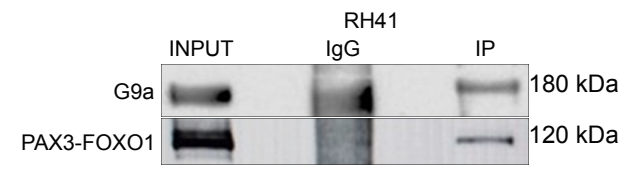

G

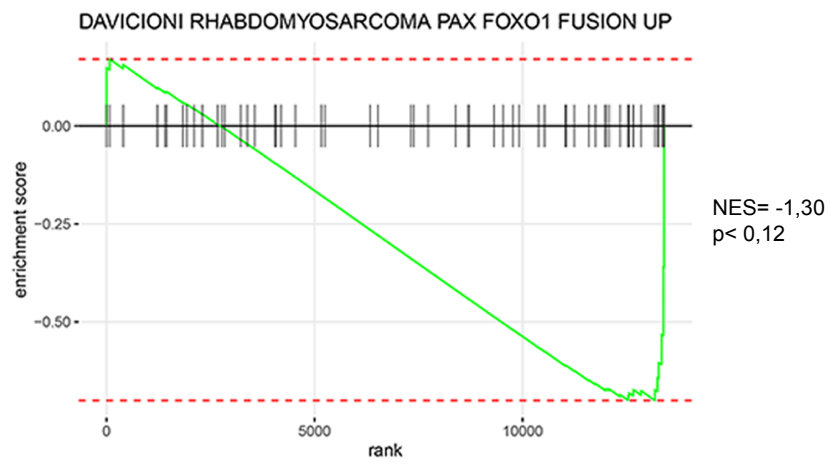

REN ALVEOLAR RHABDOMYOSARCOMA UP

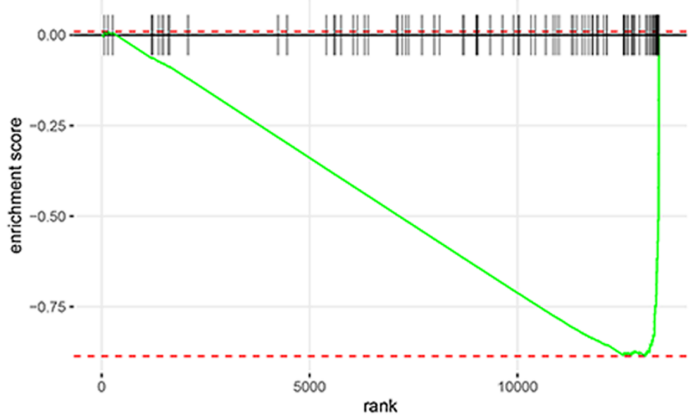

NES $=-1,72$ $\mathrm{p}<0,001$

$\mathbf{L}$

$\mathrm{RH} 41$

$\mathrm{RH} 30$

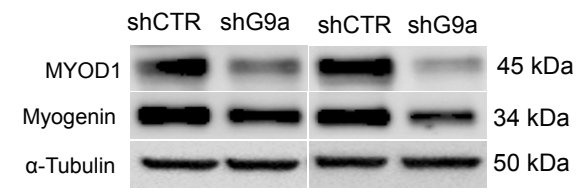

M

RH41

$\mathrm{RH} 41$

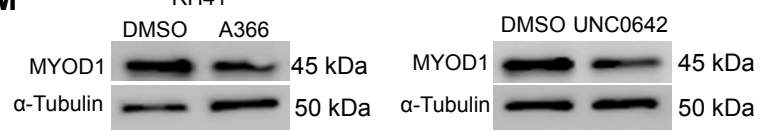

\section{Figure 3}

\section{DDX5 and G9a regulate PAX3-FOXO1 expression}

(A) Western blot analysis for DDX5 and PAX3-FOXO1 in RH30 cells treated with siCTR and siDDX5. $\alpha$-Tubulin shown as loading control. (B) Western blot for G9a and PAX3-FOXO1 in RH30 (left) and RH41 (right) cells treated with shCTR and

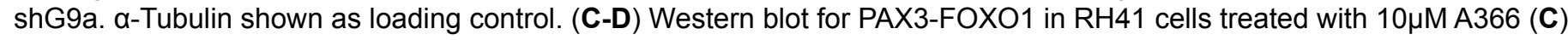
and $2 \mu \mathrm{M}$ UNC0642 (D) for six days. Control cells were treated with DMSO and all samples were normalized with $\alpha$-Tubulin. (E) Western blot analysis of G9a immunoprecipitation in RH30 (left) and RH41 (right) cells. PAX3-FOXO1 is detected in the G9a immunoprecipitates, but not in the IgG negative control. Input are shown on the left. (F) Western blot analysis for G9a and PAX3-FOXO1 in RH41 cells treated with A-366 as indicated in (C) and immunoprecipitated for G9a. IgG were used as a negative control. (G) GSEA of RNA-seq performed in shCTR and shG9a RH41 cells, on genes found upregulated by PAX3-FOXO1, using two different data sets (27) and (26). (H) Validation of the indicated PAX3-FOXO1 target genes by qRT-PCR in shCTR and shG9a cells. Transcription values were normalized to GAPDH. Graphs show the mean +/- SD from $\mathrm{n}=3$ independent experiments. (I) mRNA levels of MYOD1 and myogenin quantified by qRT-PCR after shG9a treatment in RH41 and RH30 cells. Transcription values were normalized to GAPDH. Graphs show the mean +/- SD from n=3 independent experiments. (L) Western blot analysis for MYOD1 and Myogenin in RH41 and RH30 cells after shCTR and shG9a. $\alpha$-tubulin was used for normalization. (M) Western blot of MYOD and MYOG protein levels in RH41 cells treated with $10 \mu \mathrm{M}$ A366 or with $2 \mu \mathrm{M}$ UNC0642 for six days. $\alpha$-tubulin was used for normalization. Statistical significance has been assessed in (H) and (I) by unpaired Student's t-test; $\left({ }^{*} p<0.05,{ }^{* *} p<0.01,{ }^{* * *} p<0.001\right.$, ns $\left.>0.05\right)$. 


\section{FIGURE 4}

bioRxiv preprint doi: https://doi.org/10.1101/2020.07.08.194092; this version posted July 10, 2020. The copyright holder for this preprint (which was not certified by peer review) is the author/funder, who has granted bioRxiv a license to display the preprint in perpetuity. It is

A made available under aCC-BY-NC-ND 4.0 International license.

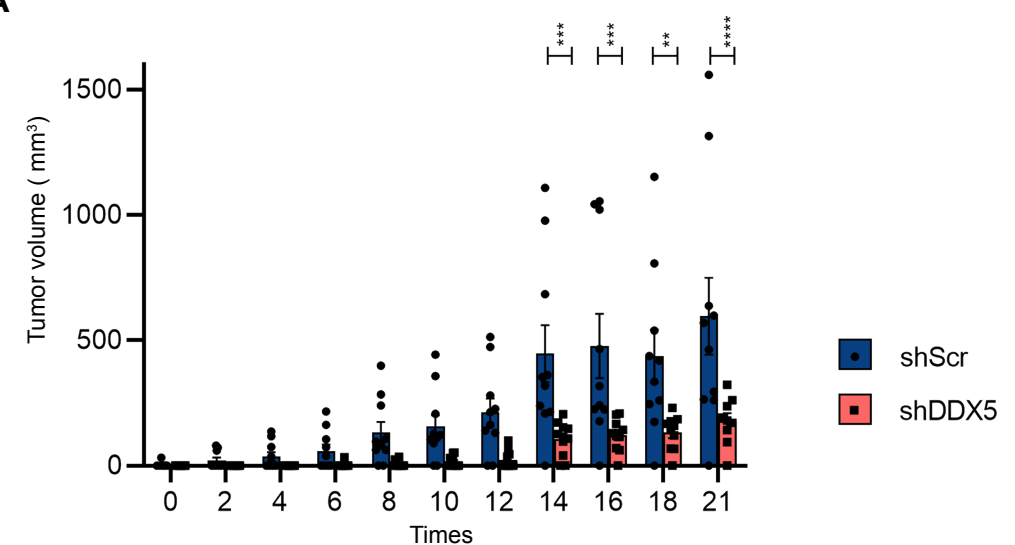

B
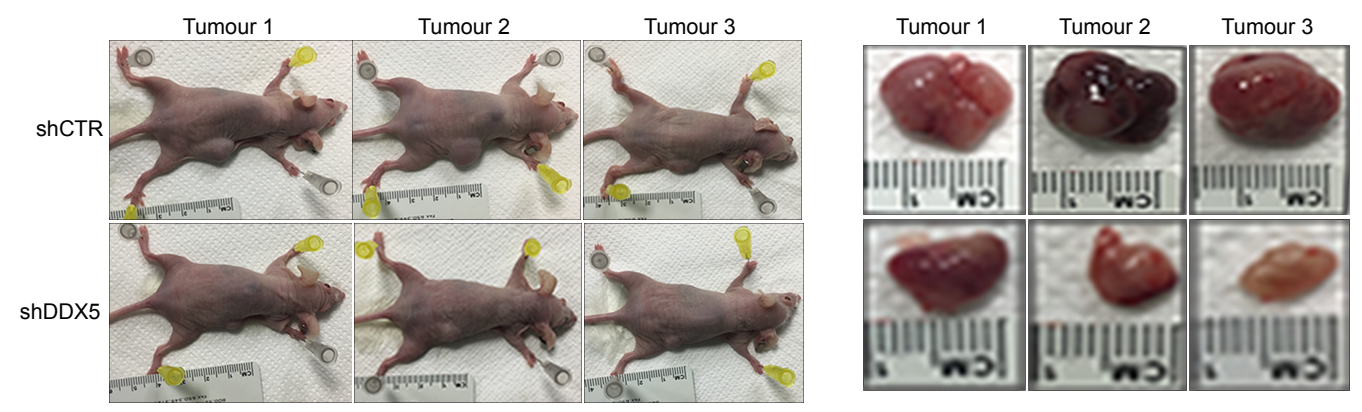

C
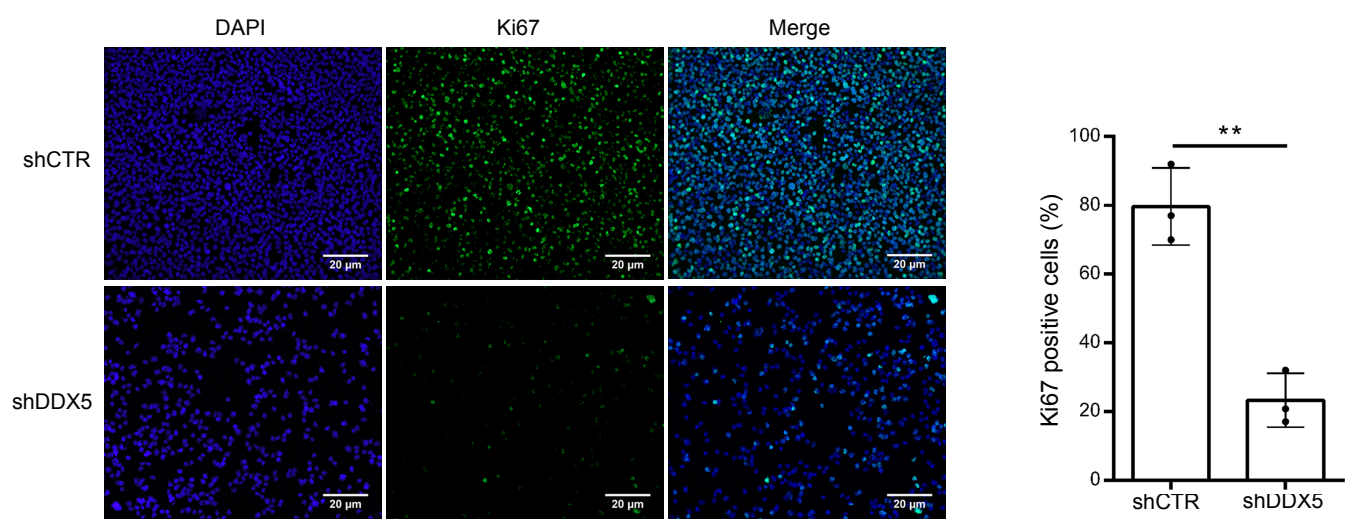

D

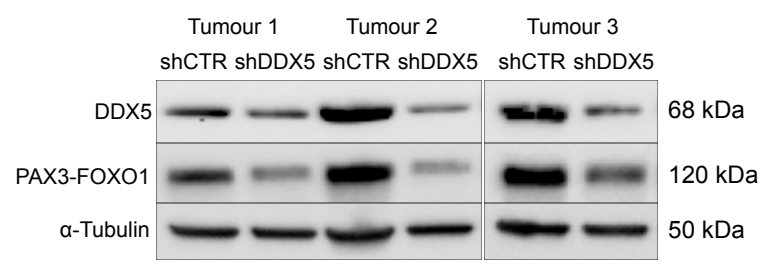

E

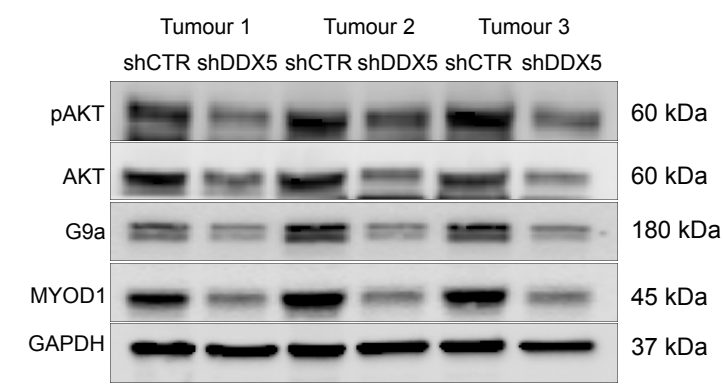

\section{Figure 4}

DDX5 promotes FP-RMS growth in vivo.

(A) Bar charts represent tumor volumes in SCTR and shDDX5 treated mice from day of injection (day 0 ) to day of tumor resection (day 21). Data are presented as mean $+/$-sem of $n=10$ mice/experimental group. Statistical significance assessed by 2 -way Anova, with Sidak's multiple comparison test. ${ }^{* *} p<0.01,{ }^{* * *} p<0.001,{ }^{* * * *} p<0.0001$. (B) Three representative shCTR and shDDX5 treated mice and matching resected tumors at day 21. (C) Representative images of Ki67 immunostaining (green) on isolated shCTR and shDDX5 tumors. DAPI (blue) was used to stain nuclei. Scale bars: 20 $\mu \mathrm{m}$. Histogram (on the right) shows quantification of the percentage of Ki67+ cells. Data are represented as mean $+/$ - sd of $n=3$ independent tumors (D-E) Western blot analysis for the indicated protein on extracts derived from $n=3$ tumors/experimental group. a-tubulin and GAPDH were used for normalization. 


\section{Supplemental Figure 1}

(which was not certified by peer review) is the author/funder, who has granted bioRxiv a license to display the preprint in perpetuity. It is made available under aCC-BY-NC-ND 4.0 International license.

A

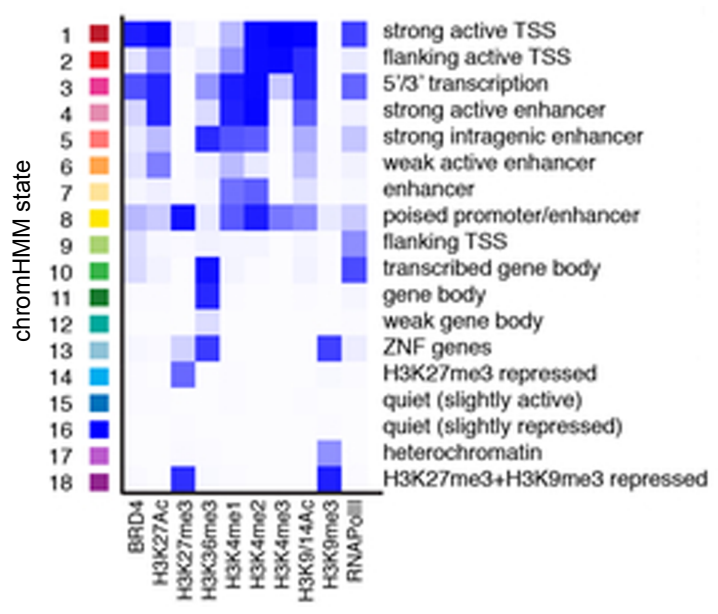

B

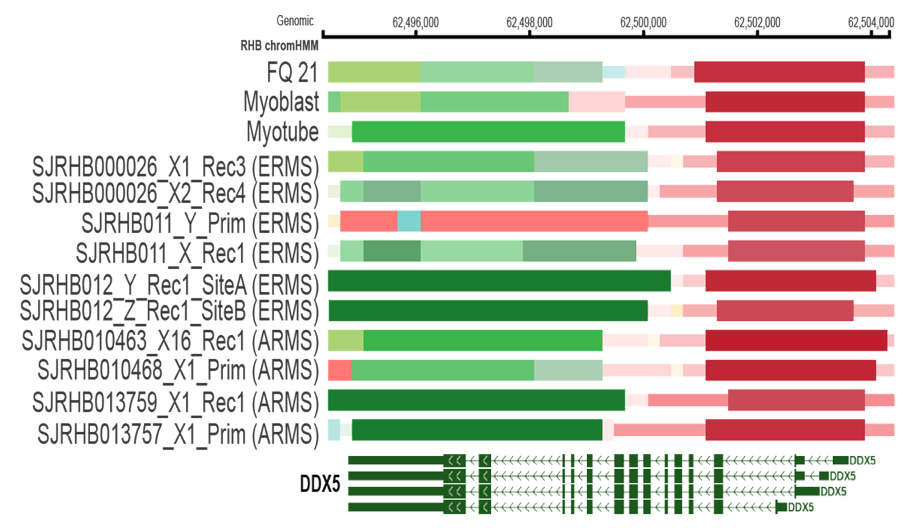

\section{Supplemental Figure 1}

(A) Legend of the 18 chromatin hidden Markov modeling (chromHMM) states (15) identified in the study (14). (B) ChromHMM state of DDX5 in RMS patient-derived xenografts (PDX) from ERMS and ARMS, as compared to normal myoblasts and myotubes. The bars show a strong active transcription start site (TSS) (red bars) and an actively transcribed gene body (green bars) in either normal myoblasts and myotubes, and primary ERMS and ARMS samples. 
SupplemewtaleFrigurentZs://doi.org/10.1101/2020.07.08.194092; this version posted July 10, 2020. The copyright holder for this preprint (which was nol certified by peer review) is the author/funder, who has granted bioRxiv a license to display the preprint in perpetuity. It is made available under aCC-BY-NC-ND 4.0 International license.

A
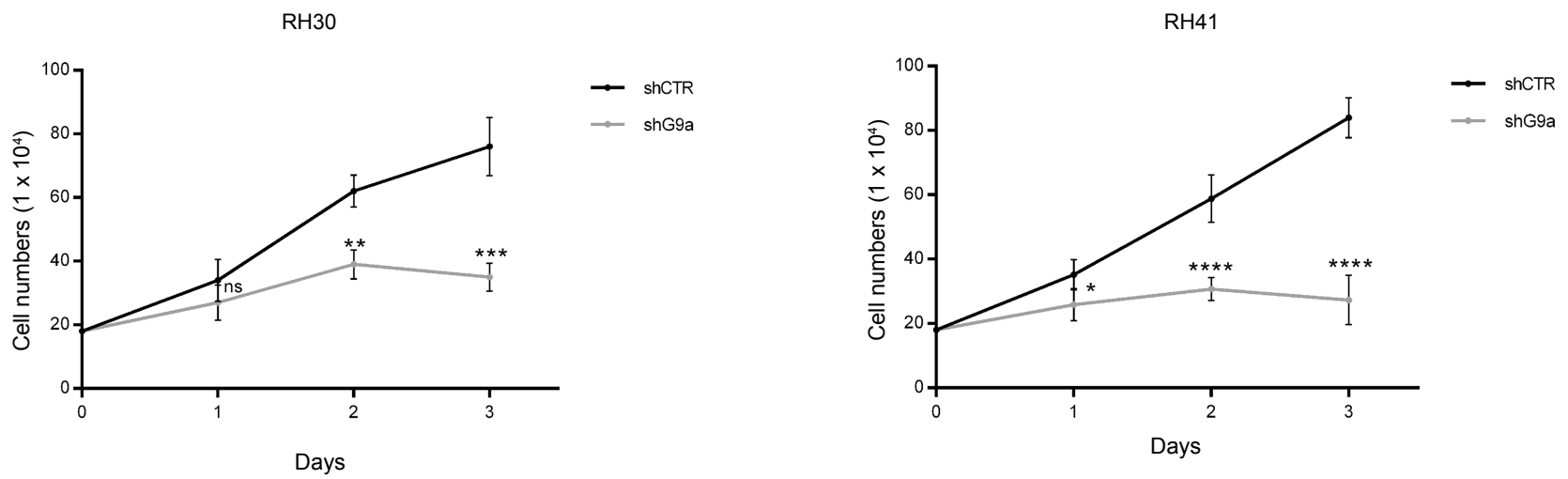

B

$\mathrm{RH} 30$

$\mathrm{RH} 41$
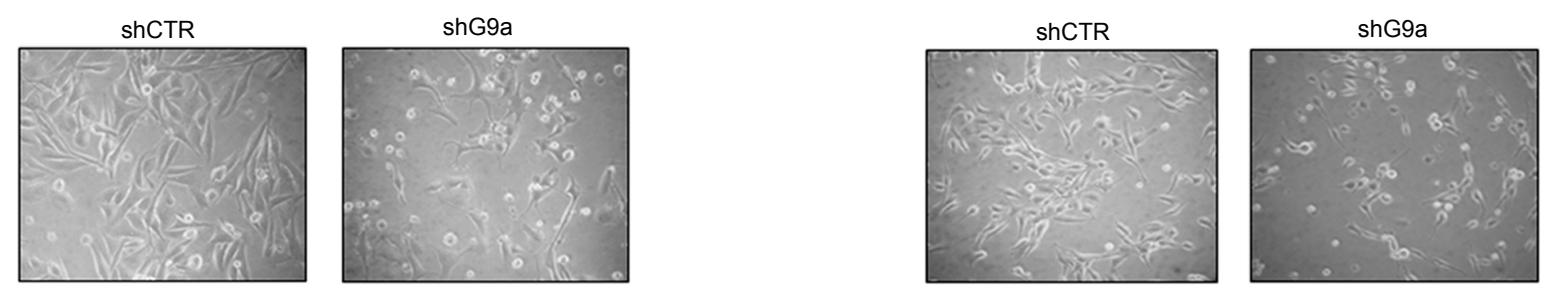

C

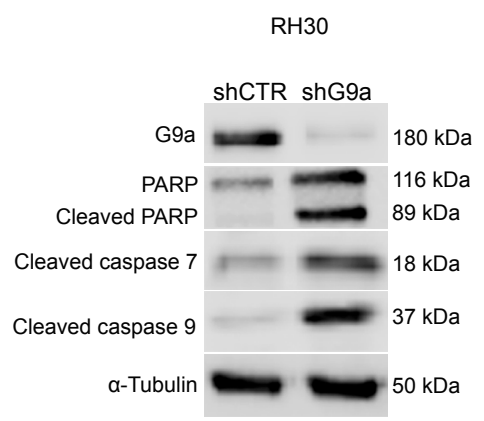

$\mathrm{RH} 41$

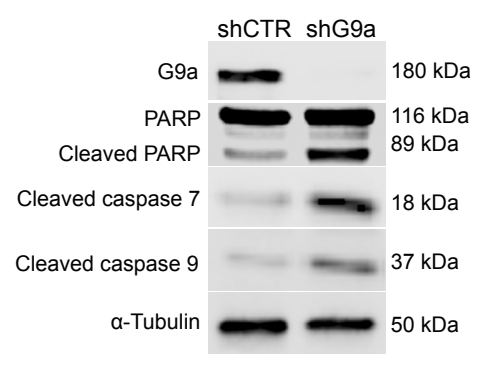

Supplemental Figure 2

(A) RH30 and RH41 cells growth curves after shCTR and shG9a treatment. Cells were counted 1, 2 and 3 days after treatment. Graphs show mean + - SD from $n=3$ independent experiments Statistical significance has been assessed by 2-way Anova, with Sidak's multiple comparison test. ${ }^{* *} p<0.01,{ }^{* * *} p<0.001,{ }^{* * * *} p<0.0001$. (B) Representative phase contrast images of RH30 (left) and RH41 (right), shCTR and shG9a, analyzed after 3 days after treatment. (C) Western blot of G9a, cleaved PARP, cleaved caspase 7 and cleaved caspase 9 from extracts of RH30 and $\mathrm{RH} 41$ cells 3 days after shG9a silencing. Normalization with $\alpha$-tubulin. 


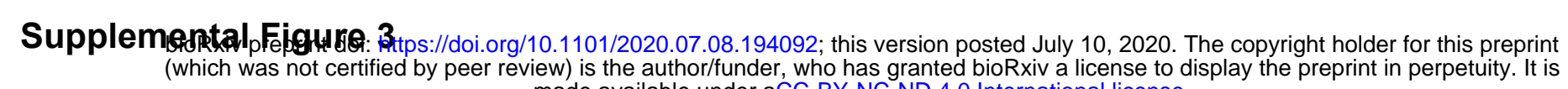
made available under aCC-BY-NC-ND 4.0 International license.

A
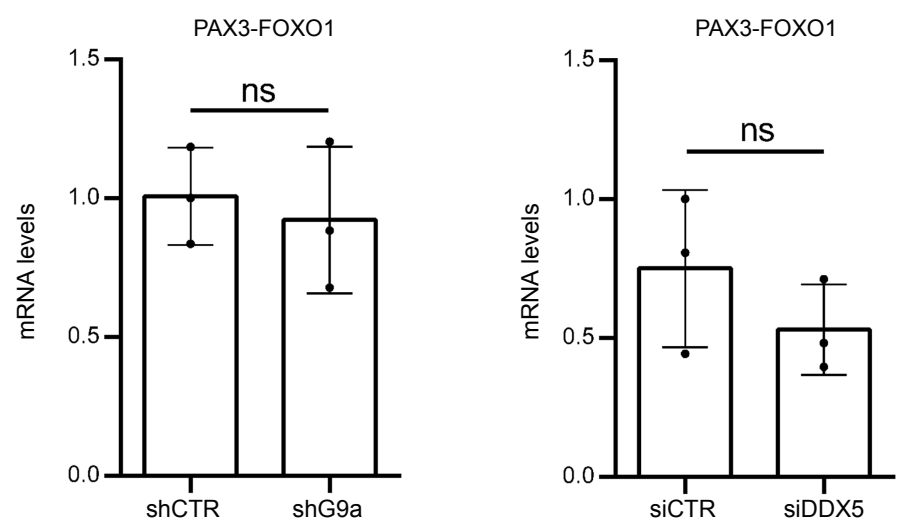

B
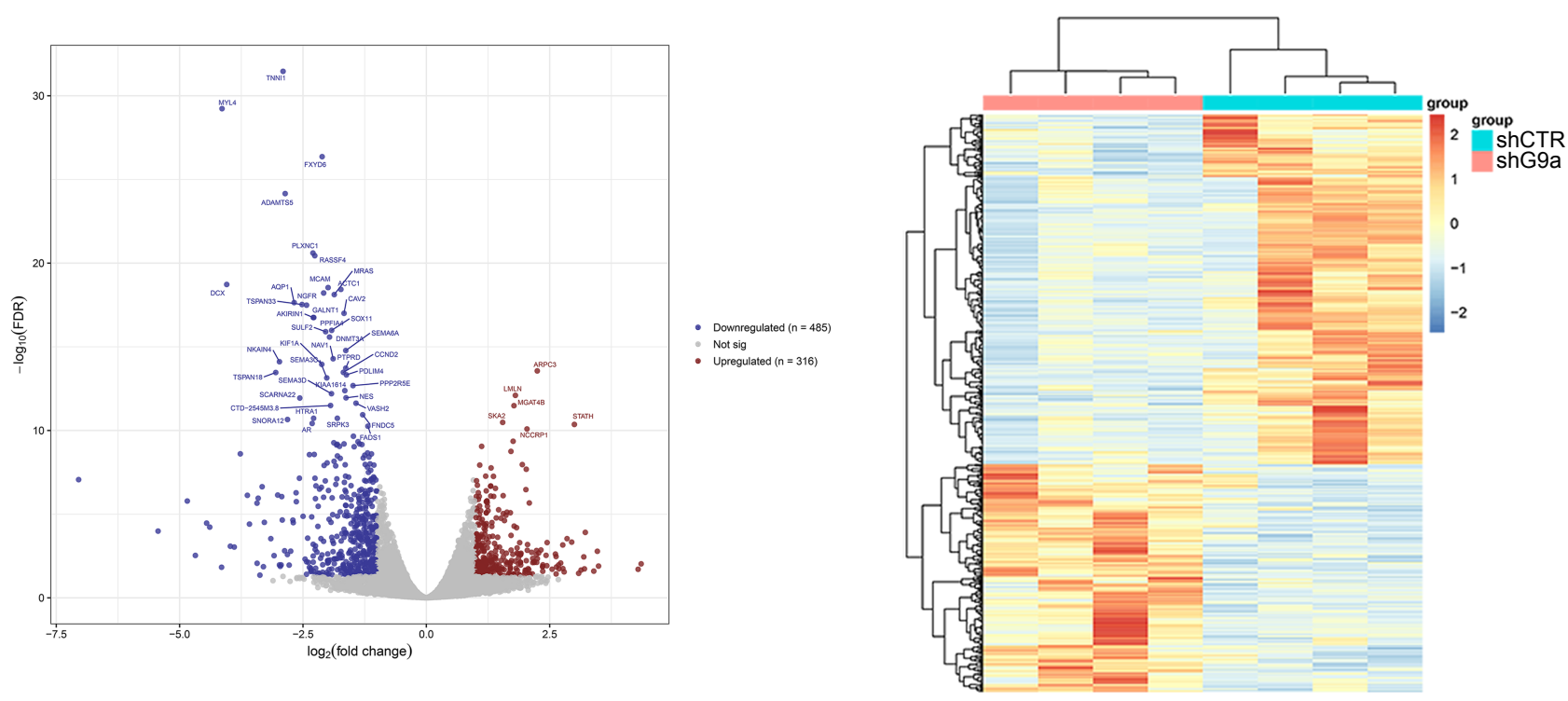

C

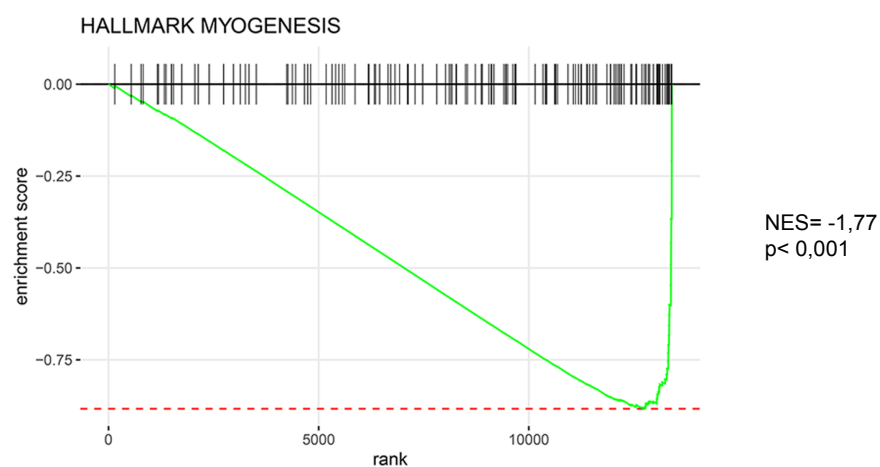

\section{Supplemental Figure 3}

(A) qRT-PCR of PAX3-FOXO1 in shG9a and siDDX5 treatments. Graphs show the mean +/-SD values from $n=3$ independent experiments. Statistical significance has been assessed by an unpaired Student's t-test; $p>0,05$ (no statistical significance, ns). (B) RNA-seq analysis after G9a knockdown. Volcano plot of shG9a vs shCTR in RH41 cells (left) showing differentially expressed genes (DEGs) (downregulated genes are highlighted in blue and upregulated genes are red). Heatmap of DEGs in RH41 cells $72 \mathrm{~h}$ after transfection (right). RNAseq analysis was performed for genes that are regulated by G9a at the transcript-level with more than two-fold regulation (Foldchange $>2$ and FDR $<0.05$ ). (C) GSEA of RNA-seq performed in shCTR and shG9a RH41 cells, on "Myogenesis" signature. 